hep-ph/9309335, RU-93-45

\title{
Naturalness Versus Supersymmetric Non-renormalization Theorems
}

\author{
Nathan Seiberg \\ Department of Physics and Astronomy \\ Rutgers University, Piscataway, NJ 08855-0849
}

\begin{abstract}
We give an intuitive proof of a new non-renormalization theorem in supersymmetric field theories. It applies both perturbatively and non-perturbatively. The superpotential is not renormalized in perturbation theory but receives non-perturbative corrections. However, these non-perturbative corrections are not generic functions of the fields consistent with the symmetries. Certain invariant terms are not generated. This violation of naturalness has applications to dynamical supersymmetry breaking.
\end{abstract}

9/93 


\section{Introduction}

The physical principle of naturalness as articulated by 'tHooft [1] states that a small parameter is natural only when a symmetry is gained as it is set to zero. In the context of quantum field theory, if a bare parameter is unnaturally set to zero, radiative corrections lead to a renormalized non-zero value. Therefore, if we want a small renormalized value without a symmetry, the bare value has to be fine tuned. The apparent violation of this principle in the value of the Higgs mass is known as "the gauge hierarchy problem" (a more dramatic fine tuning problem is that of the cosmological constant).

The main motivation to study low-energy supersymmetry is that it can provide a solution to the gauge hierarchy problem. In supersymmetric theories the parameters in the superpotential are subject to a weaker form of naturalness. Because of the perturbative non-renormalization theorems [2], the superpotential is not renormalized in perturbation theory. Therefore, if for some reason the Higgs mass is very small or zero at tree levelli, it remains so to all orders even when it is not protected by a symmetry.

The known proof [2] of this non-renormalization theorem depends on the details of perturbation theory and supergraph techniques. One of the results of this paper is a more intuitive derivation of this theorem.

Clearly, we would like supersymmetry to be broken non-perturbatively. This is possible only if the non-renormalization theorem is violated beyond perturbation theory in four dimensions. Indeed, it was shown in [3] that once non-perturbative effects are taken into account new terms can appear in the superpotential.

The obvious question is then: is the exact superpotential a generic function of the fields consistent with the symmetries? In other words, is the full strength of the naturalness principle applicable non-perturbatively? As we will show, the answer to this question is negative. There are powerful non-renormalization theorems which are valid in the full, non-perturbative theory.

In section 2 we review some of the non-perturbative techniques in supersymmetric gauge theories with matter. We will follow the point of view of [3] and [4] (for other ap-

1 Such a situation is common in string models where bare mass terms can be absent at tree level without a symmetry. 
proaches to the study of these theories see [5] and [6]). In section 3 we present our new non-renormalization theorem. Section 4 is devoted to various examples and applications. We rederive the standard perturbative non-renormalization theorem in Wess-Zumino models and extend it beyond perturbation theory, we also explain some of the results of section 2 and use the new theorem to solve some problems in models with dynamical supersymmetry breaking.

\section{Examples of non-perturbative effects in SUSY theories}

\subsection{Examples of non-perturbative effects in gauge theories}

We consider an $S U(N)$ gauge theory with $N_{f}$ flavors of quark superfields $Q$ and $\bar{Q}$ transforming as $N$ and $\bar{N}$ under the gauge group. We first assume that the quarks are massless and the superpotential vanishes.

An important property of this theory is the existence of classical "flat directions." There is a continuum of inequivalent classical ground states labeled by the expectation values of $Q$ and $\bar{Q}$. These expectation values break the gauge symmetry. Taking loop corrections into account, the coupling constant of the theory depends on $\langle Q\rangle$ and $\langle\bar{Q}\rangle$ and the theory becomes weakly coupled far out along the flat directions.

The classical theory is invariant under the global symmetry

$$
U\left(N_{f}\right)_{L} \times U\left(N_{f}\right)_{R} \times U(1)_{R}
$$

where the last factor is an $\mathrm{R}$ symmetry. This symmetry is valid to all orders in perturbation theory and prevents any superpotential from being generated. Therefore, in this case the perturbative non-renormalization theorem is perfectly natural; it follows from a symmetry. The large classical vacuum degeneracy mentioned above is not lifted in perturbation theory.

Non-perturbatively, the axial $U(1)$ in (2.1) is explicitly broken and a unique term is allowed by the unbroken symmetry [7]

$$
\frac{\Lambda^{\frac{3 N-N_{f}}{N-N_{f}}}}{(\operatorname{det} \bar{Q} Q)^{\frac{1}{N-N_{f}}}}
$$


where the exponent of the determinant in flavor space is determined by the $\mathrm{R}$ symmetry and the power of the scale of the theory, $\Lambda$, is determined on dimensional grounds. This term exists only for $N>N_{f}$. The analysis of reference [3] shows that the term (2.2) is indeed generated. For $N_{f}=N-1$ it is generated by instantons and for $N_{f}<N-1$ it is generated by other non-perturbative effects. Since the coupling constant depends on the fields and the field dependence in (2.2) is uniquely determined by the symmetries, the approximate results of these non-perturbative calculations are exact.

The conclusion is that the non-renormalization theorem is violated non-perturbatively. Furthermore, the effective Lagrangian is the most general one consistent with the symmetries. Therefore, we might be tempted to guess that the full strength of naturalness applies non-perturbatively. This guess is incorrect!

\subsection{Examples of non-perturbative effects depending on couplings in the superpotential}

The potential derived from equation (2.2) slopes to zero at infinity and the massless theory does not have a ground state. To stabilize the potential we can add mass terms

$$
\sum_{i} m_{i} \bar{Q}_{i} Q^{i}
$$

to the superpotential. For small $m_{i}$ the effective superpotential is the sum of the dynamically generated term (2.2) and the tree level terms (2.3). When the $m_{i}$ 's are not small there might be higher order corrections given by powers of $m_{i}$. We now study these corrections in the simple case where only one mass $m=m_{N_{f}}$ is non-zero. We would like to integrate out the massive quark and to study an effective Lagrangian for the light quarks. We study two different limits:

1. $m \gg \Lambda$. Below the scale $m$ we have an $S U(N)$ gauge theory with $N_{f}-1$ quarks. The scale of the low-energy theory $\Lambda_{L}$ is different than $\Lambda$ because only $N_{f}-1$ quarks contribute to the running of the coupling constant below $m$. We find

$$
\Lambda_{L}=\left(m \Lambda^{3 N-N_{f}}\right)^{\frac{1}{3 N-N_{f}+1}}
$$

Using the superpotential (2.2) with this scale and $N_{f}-1$ flavors we find

$$
W_{e f f} \sim \frac{\left(m \Lambda^{3 N-N_{f}}\right)^{\frac{1}{N-N_{f}+1}}}{\left(\operatorname{det}^{\prime} \bar{Q} Q\right)^{\frac{1}{N-N_{f}+1}}}
$$


where $\operatorname{det}^{\prime} \bar{Q} Q$ denotes a determinant over the light fields.

2. $m \ll \Lambda$. We start by analyzing the case of $N_{f}=N$. Repeating the instanton analysis of [3] we find that the massless theory has four fermion zero modes. Therefore no superpotential can be generated. This is consistent with the symmetries of the massless theory. For small $m$ two of the fermion zero modes can be lifted at order $m$ and generate the superpotential

$$
W_{e f f} \sim \frac{m \Lambda^{2 N}}{\operatorname{det}^{\prime} \bar{Q} Q}
$$

For $N_{f}<N$ the dynamics of the massive quarks $Q_{N_{f}}$ and $\bar{Q}_{N_{f}}$ are determining by analyzing the superpotential

$$
\frac{\Lambda^{\frac{3 N-N_{f}}{N-N_{f}}}}{(\operatorname{det} \bar{Q} Q)^{\frac{1}{N-N_{f}}}}+m \bar{Q}_{N_{f}} Q_{N_{f}}
$$

The equation of motion of the massive quarks leads to

$$
\left\langle\bar{Q}_{N_{f}} Q_{N_{f}}\right\rangle \sim \frac{\Lambda^{\frac{3 N-N_{f}}{N-N_{f}+1}}}{m^{\frac{N-N_{f}}{N-N_{f}+1}}\left(\operatorname{det}^{\prime} \bar{Q} Q\right)^{\frac{1}{N-N_{f}+1}}}
$$

and to an effective superpotential

$$
W_{e f f} \sim \frac{\left(m \Lambda^{3 N-N_{f}}\right)^{\frac{1}{N-N_{f}+1}}}{\left(\operatorname{det}^{\prime} \bar{Q} Q\right)^{\frac{1}{N-N_{f}+1}}}
$$

We see that the $m$ dependence in these two limits is the same. This strongly suggests that it is exact and that the effective superpotential does not receive higher order corrections in $m$.

\section{New non-renormalization theorem}

The following discussion is a generalization of an unpublished argument [8] for the perturbative non-renormalization theorem of the superpotential, $W$. It was motivated by a similar argument in string theory [9]. In string theory the coupling constant is a dynamical field - the dilaton. Its superpartner is an axion and therefore to all orders in perturbation 
theory, the theory is constrained by a Peccei-Quinn symmetry. The combination of this symmetry with the holomorphicity of $W$ leads to the lack of renormalization of $W$ in perturbation theory.

In the same spirit, we now think of all coupling constants in the superpotential, $\lambda_{i}$, as background chiral field\$2 2 . The effective superpotential $W_{\text {eff }}$ of the dynamical fields $\phi_{I}$ and the background fields $\lambda_{i}$ is subject to the following constraints:

1. Symmetry. For $\lambda_{i}=0$ the theory has a large global symmetry group $G$. This symmetry is explicitly broken by the couplings $\lambda_{i}$. When we think of $\lambda_{i}$ as fields, we can interpret their non-zero values as spontaneously breaking $G$. Then, the effective Lagrangian which depends both $\phi_{I}$ and $\lambda_{i}$ should be invariant under $G$. Similar constraints in situations of explicitly broken symmetries are common in physics and are known as selection rules.

2. Holomorphicity. The effective superpotential, $W_{e f f}$, is a (locally) holomorphic function of the fields. Since we treat $\lambda_{i}$ as fields, $W_{\text {eff }}$ is independent of $\lambda_{i}^{\dagger}$. This is unlike the situation in ordinary field theories where the effective potential depends both on $\lambda$ and on $\lambda^{\dagger}$.

3. Asymptotic freedom of gauge couplings. The effective superpotential can depend on the dynamically generated scale of the theory $\Lambda$. In perturbation theory there might be factors of $\log \Lambda$ which for simplicity we will ignore. Non-perturbatively there are powers of $\Lambda$. It is obvious that $W_{\text {eff }}$ should be smooth in the limit $\Lambda \rightarrow 0$; i.e. there are no negative powers of $\Lambda$. In most cases this means that $W_{\text {eff }}$ cannot grow faster than $\phi^{3}$ as a field $\phi \rightarrow \infty$. Furthermore, when there are no strongly coupled sectors - there are no unbroken non-Abelian gauge groups, the leading non-perturbative effect is given by instantons. Then, the power of $\Lambda$ cannot be smaller than in $\exp \left(-\frac{8 \pi^{2}}{g(\mu)^{2}}\right)=\left(\frac{\Lambda}{\mu}\right)^{x}$ where $x$ is determined by the one loop beta function (e.g. for an $S U(N)$ gauge theory with $N_{f}$ flavors in the fundamental representation $x=3 N-N_{f}$ ).

4. Weak coupling. The behavior of $W_{e f f}$ as the coupling constants in the bare super-

2 These can be thought of as dynamical fields whose kinetic terms have infinite coefficients. Since these are classical fields, their dimensions are the same as the dimensions of the coupling constants and the theory is renormalizable. 
potential $\lambda_{i} \rightarrow 0$ can often be analyzed perturbatively, thus constraining the small $\lambda_{i}$ limit. Sometimes there are more light fields at $\lambda_{i}=0$ than at non-zero $\lambda_{i}$. When these fields are integrated out and are not included in the effective action, $W_{\text {eff }}$ might be non-analytic at $\lambda_{i}=0$.

As we will see in the examples in the next section, the combination of these constraints is extremely powerful and leads to many results. It is easy to rederive the standard perturbative non-renormalization theorem. It is also easy to see when it can be violated (e.g. as in the examples in section 2). Finally, one can derive a number of exact results which go beyond any approximation scheme. These exact results show that $W_{\text {eff }}$ is not a generic function of the fields consistent with the symmetries. Some terms which are consistent with all the symmetries in the problem are not generated either perturbatively or non-perturbatively. This is in clear violation of the principle of naturalness.

We should caution the reader that our arguments are somewhat heuristic. We assume that the theories exist as quantum field theories and that there are no unexpected anomalies in supersymmetry (or in any other global symmetry). In other words, we assume that the theory can be regularized while preserving all these symmetries.

The effective action we discuss is the Wilsonian effective action. It should be distinguished from the 1PI effective action. The latter suffers from IR ambiguities. The Wilsonian effective action at a scale $\mu$ is obtained by integrating out all fields whose mass is larger than $\mu$ and the high momentum $(p>\mu)$ modes of the light fields. It does not suffer from any IR ambiguities. The distinction between the Wilsonian effective action and the 1PI effective action is particularly important when massless particles are present. Then, IR subtleties can lead to "holomorphic anomalies" in the 1PI effective action. However, these cannot appear in the Wilsonian effective action. This distinction was made clear in [10] where some confusions associated with another holomorphic function - the coefficient of $W_{\alpha}^{2}-$ were clarified. Similarly, the non-holomorphic threshold contributions to this function [11] and the holomorphic anomalies of [12] are associated with IR properties of the 1 PI effective action. 


\section{Examples}

\subsection{Wess-Zumino models}

The superpotential is

$$
W_{\text {tree }}=m \phi^{2}+\lambda \phi^{3}
$$

For $m=\lambda=0$ the theory has a $U(1) \times U(1)_{R}$ global symmetry (the second factor is an R symmetry). The field $\phi$ transforms as $(1,1)$ under the symmetry. The couplings in (4.1) determine the charges of $m$ to be $(-2,0)$ and of $\lambda$ to be $(-3,-1)$. The most general renormalized superpotential invariant under $U(1) \times U(1)_{R}$ is

$$
W_{e f f}=m \phi^{2} f\left(\frac{\lambda \phi}{m}\right)
$$

where $f$ is an arbitrary holomorphic function. Consider the coefficient of $\phi^{n}$. From (4.2) it is of the form $\lambda^{n-2} \frac{1}{m^{n-3}} \phi^{n}$. This is exactly the answer from a tree graph with $\phi$ exchanges. Its contribution should not be included either in the Wilsonian or in the 1PI effective action. Higher order corrections in $\lambda$ to the coefficient of $\phi^{n}$ are not compatible with the form (4.2). Therefore, the effective action must be

$$
W_{\text {eff }}=m \phi^{2}+\lambda \phi^{3}=W_{\text {tree }} .
$$

We conclude that the tree level superpotential is not renormalized, in accord with the standard non-renormalization theorems [2]. The "holomorphic anomaly" in $W$ which was found in 13 is of the form $\lambda^{3} \lambda^{\dagger 2} \phi^{3}$. It exists only in the 1PI effective action of the $m=0$ theory and clearly arises from IR problems. We should add that strictly speaking this theory is not expected to exist non-perturbatively because it is not asymptotically free.

\subsection{SUSY QCD with a massive quark}

This theory was studied in subsection 2.2. Without the mass term the theory is invariant under the group

$$
G=S U\left(N_{f}\right)_{L} \times S U\left(N_{f}\right)_{R} \times U(1)_{V} \times U(1)_{R}
$$


where the last factor is an $\mathrm{R}$ symmetry. The mass term for the last quark breaks it to

$$
H=S U\left(N_{f}-1\right)_{L} \times S U\left(N_{f}-1\right)_{R} \times U(1)_{V} \times U(1)_{V^{\prime}} \times U(1)_{R} .
$$

The mass term is in the $\left(N_{f}, N_{f}\right)$ representation of $G$. $H$ invariance and dimensional analysis constrain the low-energy superpotential to be of the form

$$
\Lambda^{\frac{3 N-N_{f}+1}{N-N_{f}+1}} f(m / \Lambda) \frac{1}{\left(\operatorname{det}^{\prime} \bar{Q} Q\right)^{\frac{1}{N-N_{f}+1}}}
$$

where det $^{\prime}$ is a determinant over the $S U\left(N_{f}-1\right)_{L} \times S U\left(N_{f}-1\right)_{R}$ indices of the light fields. $G$ invariance can be used to fix the $m$ dependence of the function $f$. It is enough to look at the axial $U(1)$ symmetry under which all the light quarks have charge 1 and the massive quark has charge $-N_{f}+1$. Clearly, $m$ has charge $2 N_{f}-2$. Therefore, the superpotential is invariant only for $f \sim\left(\frac{m}{\Lambda}\right)^{\frac{1}{N-N_{f}+1}}$ and

$$
W_{e f f} \sim \frac{\left(m \Lambda^{3 N-N_{f}}\right)^{\frac{1}{N-N_{f}+1}}}{\left(\operatorname{det}^{\prime} \bar{Q} Q\right)^{\frac{1}{N-N_{f}+1}}} .
$$

The non-analyticity at $m=0$ arises from integrating out one of the quarks which is massless at that point (see constraint 4 in section 3 ).

This proves that expressions (2.9) and (2.5) are indeed exact and explains why we found the same answer in the small and in the large $m$ limits.

\section{3. $S U S Y Q C D$ with a superpotential $S \bar{Q} Q+\lambda^{\prime} S^{3}$}

We now study SUSY QCD with a coupling to a gauge singlet $S$ through the superpotential [3]

$$
W_{\text {tree }}=\lambda S \sum \bar{Q} Q+\lambda^{\prime} S^{3}
$$

where the sum in the first term is both over color and flavor indices. For $\lambda=\lambda^{\prime}=0$ the theory is invariant under

$$
G=S U\left(N_{f}\right)_{L} \times S U\left(N_{f}\right)_{R} \times U(1)_{V} \times U(1)_{S} \times U(1)_{R}
$$

where $U(1)_{S}$ acts only on $S$ and $U(1)_{R}$ is an R symmetry under which $S$ has charge zero and $\bar{Q} Q$ has charge $2 \frac{N_{f}-N}{N_{f}}$ (actually, since $S$ is a decoupled free field there are more 
symmetries). These two $U(1)$ symmetries are explicitly broken by the interactions in (4.8).

Thinking of the couplings as fields we can assign to $\lambda$ charges $\left(-1,2 \frac{N}{N_{f}}\right)$ and to $\lambda^{\prime}$ charges $(-3,2)$. For $\lambda^{\prime}=0$ but non-zero $\lambda$ the theory has a flat direction labeled by the expectation value of $S$. The coupling $\lambda$ leads to masses for the quarks and they can be integrated out. The low-energy effective Lagrangian depends then only on $S$. Using the symmetries and dimensional analysis the superpotential has the form

$$
W_{e f f}=\lambda^{\prime} S^{3} f\left(\frac{\Lambda^{\frac{3 N-N_{f}}{N}}(\lambda S)^{\frac{N_{f}}{N}}}{\lambda^{\prime} S^{3}}\right)
$$

where $\Lambda$ is the dynamically generated scale of the gauge theory. By asymptotic freedom (constraint 3 in section 3) $f(x)$ is regular at $x \rightarrow 0$. From the behavior as $\lambda^{\prime} \rightarrow 0$ (constraint 4 in section 3$), f(x)=\alpha x+1+$ smaller terms ( $\alpha$ is a constant) as $x \rightarrow \infty$. This fixes the exact superpotential to be

$$
W_{e f f}=\lambda^{\prime} S^{3}+\alpha \Lambda^{\frac{3 N-N_{f}}{N}}(\lambda S)^{\frac{N_{f}}{N}}
$$

The second term is generated non-perturbatively [4]. (The non analyticity at $\lambda=0$ arises from integrating out fields $(Q$ and $\bar{Q})$ which are massless at that point.) This example (for $\left.N_{f} \leq 3 N\right)$ demonstrates that in an asymptotically free theory $W_{\text {eff }}$ can grow at large field strength but not faster than $S^{3}$. Since (4.11) is exact, we learn that there are no higher order corrections in $\lambda$ or $\lambda^{\prime}$. Furthermore, although all powers of $S$ are consistent with the symmetries of the theory, only one of them is dynamically generated. The superpotential (4.11) leads to a supersymmetric minimum at finite $S$.

\subsection{Dynamical SUSY breaking without $R$ symmetry}

The fact that the perturbative non-renormalization theorem can be violated nonperturbatively opens the way to dynamical supersymmetry breaking. As advocated by Witten [14], the gauge hierarchy problem can be solved in a theory where supersymmetry is unbroken at tree level (and therefore to all orders in perturbation theory), but a tiny nonperturbative effects breaks it. Such a scenario can naturally generate the weak scale much below the Planck scale. Models with dynamical supersymmetry breaking were studied 
in [4] and [6]. Recently, it was pointed out [15] that supersymmetry breaking can take place only in theories with a continuous $\mathrm{R}$ symmetry provided two conditions are satisfied. The conditions are: (1) the breaking of supersymmetry can be described by a low-energy Wess-Zumino model without gauge fields and (2) the superpotential of that model is a generic function of the fields consistent with the symmetries (naturalness). This leads to a phenomenological problem because it is clear that Nature does not have such a symmetry (whether broken or not). Fortunately, our non-renormalization theorem shows that the lowenergy superpotential does not have to be generic. Therefore, we can have supersymmetry breaking without an R symmetry 15.

To demonstrate this we consider an $S U(3) \times S U(2)$ theory [15] with chiral fields transforming like

$$
\begin{array}{cccccc}
Q & \bar{d} & \bar{u} & \bar{s} & s & L \\
(3,2) & (\overline{3}, 1) & (\overline{3}, 1) & (\overline{3}, 1) & (3,1) & (1,2)
\end{array}
$$

(unlike the fields in the Standard Model this $s$ is an $S U(2)$ singlet). We impose a global $U(1)$ symmetry "hypercharge." The most general renormalizable tree level superpotential invariant under hypercharge is

$$
W_{\text {tree }}=\lambda_{d} L Q \bar{d}+\lambda_{s} L Q \bar{s}+\lambda Q^{2} s+\bar{\lambda} \bar{d} \bar{u} \bar{s}+m \bar{s} s
$$

When all Yukawa couplings are set to zero and the $S U(2)$ coupling is turned off, this model is equivalent to the example in subsections 2.2 and 4.2 with $N=N_{f}=3$ and a single massive quark. The potential has flat directions and $S U(3)$ instantons generate the term

$$
W_{\text {instanton }}=\alpha \frac{m \Lambda_{3}^{6}}{Q^{2} \bar{u} \bar{d}}
$$

for some computable constant $\alpha$.

We now turn on the $S U(2)$ gauge interactions. There are still flat directions and the low-energy theory along them is described by the three gauge invariant chiral superfields [4]

$$
\begin{aligned}
X & =L Q \bar{u} \\
Y & =L Q \bar{d} \\
Z & =Q^{2} \bar{u} \bar{d} .
\end{aligned}
$$


Turning on the Yukawa couplings, integrating $s$ and $\bar{s}$ out at tree level and adding the instanton contribution (4.14), the low-energy effective superpotential is

$$
W_{e f f}=\lambda_{d} Y+\frac{\lambda \bar{\lambda}}{m} Z+\alpha \frac{m \Lambda^{6}}{Z}
$$

This superpotential leads to supersymmetry breaking. This can be seen either in the low-energy effective theory $\left(\frac{\partial W_{\text {eff }}}{\partial Y} \neq 0\right)$ or in the full theory [15].

For $\lambda=\bar{\lambda}=0$ the model has an $\mathrm{R}$ symmetry and no flat directions. Therefore, the fact that it breaks supersymmetry is consistent with the general analysis of references [4] and [15]. However, with non-zero $\lambda$ and $\bar{\lambda}$ there is no R symmetry but supersymmetry is still broken. This is not in contradiction with the discussion in [15] because $W_{\text {eff }}$ is not a generic function invariant under the symmetries. If, for example, an invariant term like $\frac{\Lambda^{6}}{L Q d}=\frac{\Lambda^{6}}{Y}$ is dynamically generated, supersymmetry is not broken. Therefore, to establish that supersymmetry is indeed broken we must show that (4.16) is exact.

Using the symmetries of the theory without the superpotential (4.13) we find that the most general invariant function of the fields $X, Y$ and $Z$ and the coupling constants is

$$
W_{e f f}=\lambda_{d} Y f\left(\frac{\lambda \bar{\lambda} Z}{m \lambda_{d} Y}, \frac{m \Lambda^{6}}{\lambda_{d} Y Z}, \frac{\lambda_{s} \lambda \Lambda^{4}}{m \lambda_{d} Y}\right) .
$$

The dependence of $f(u, v, w)$ on $v$ represents the explicit breaking of a $U(1)$ symmetry by the $S U(3)$ anomaly and the dependence on $w$ represents the breaking of another $U(1)$ symmetry by the $S U(2)$ anomaly. The other constraints in section 3 fix the function $f(u, v, w)$ to be of the form

$$
f(u, v, w)=1+u+\alpha v+\beta w
$$

for some constants $\alpha$ and $\beta$. The last term is independent of the fields and therefore can be ignored in global supersymmetry 3 . Therefore, there are no new terms beyond those we studied. We conclude that the superpotential is not generic and SUSY is broken.

3 A constant in the superpotential is important in local supersymmetry. It is easy to check that this term has the correct quantum numbers to be generated by $S U(2)$ instantons and hence if $\beta$ is non-zero, it is proportional to $\exp \left(-\frac{8 \pi^{2}}{g_{2}^{2}(\Lambda)}\right)$. The combination $\Lambda^{4} \exp \left(-\frac{8 \pi^{2}}{g_{2}^{2}(\Lambda)}\right)$ is independent of $\Lambda$ and depends only on the scale of the $S U(2)$ theory. This is precisely the behavior expected in a contribution of an $S U(2)$ instanton. Therefore, we conjecture that an instanton calculation leads to non-zero $\beta$. 


\section{Acknowledgements}

It is a pleasure to thank T. Banks, J. Polchinski, E. Witten, and especially A. Nelson and S. Shenker for several useful discussions. This work was supported in part by DOE grant \#DE-FG05-90ER40559. 


\section{References}

[1] G. 't Hooft, in Recent Developments in Gauge Theories, ed. G. 't Hooft et.al., (Plenum, New York, 1980)

[2] M.T. Grisaru, W. Siegel and M. Rocek, Nucl. Phys. B159 (1979) 429

[3] I. Affleck, M. Dine, and N. Seiberg, Phys. Rev. Lett. 51 (1983) 1026; Nucl. Phys. B241 (1984) 493

[4] I. Affleck, M. Dine and N. Seiberg, Phys. Lett. 137B (1984) 187; Phys. Rev. Lett. 52 (1984) 493; Phys. Lett. 140B (1984) 59; Nucl. Phys. B256 (1985) 557

[5] G. Veneziano and S. Yankielowicz, Phys. Lett. 113B (1982) 321; T.R. Taylor, G. Veneziano, and S. Yankielowicz, Nucl. Phys. B218 (1983) 493; V.A. Novikov, M.A. Shifman, A. I. Vainstain and V. I. Zakharov, Nucl. Phys. B223 (1983) 445; M. Peskin, in Problems in Unification and Supergravity, ed. G. Farrar and F. Henyey (AIP, New York, 1984)

[6] G.C. Rossi and G. Veneziano, Phys. Lett. 138B (1984) 195; Y. Meurice and G. Veneziano, Phys. Lett. 141B (1984) 69; D. Amati, G.C. Rossi and G. Veneziano, Phys. Lett. 138B (1984) 195; D. Amati, K. Konishi, Y. Meurice, G.C. Rossi and G. Veneziano, Phys. Rep. 162 (1988) 169

[7] A.C. Davis, M. Dine and N. Seiberg, Phys. Lett. 125B (1983) 487

[8] J. Polchinski and N. Seiberg, (1988) unpublished

[9] M. Dine and N. Seiberg, Phys. Rev. Lett. 57 (1986) 2625

[10] M.A. Shifman and A.I Vainshtein, Nucl. Phys. B277 (1986) 456

[11] L.J. Dixon, V.S. Kaplunovsky and J. Louis, Nucl. Phys. B355 (1991) 649

[12] M. Bershadsky, S. Cecotti, H. Ooguri and C. Vafa, HUTP-93-A008, hep-th/9302103

[13] I. Jack, D.R.T. Jones and P. West, Phys. Lett. 258B (1991) 382

[14] E. Witten, Nucl. Phys. B188 (1981) 513; Nucl. Phys. B202 (1982) 253

[15] A. Nelson and N. Seiberg, UCSD/PTH 93-27, RU-93-42, hep-ph/9309299 